\title{
Using the CLOX drawing task to educate the public of the effects of hearing loss
}

Sandra C M Quinn, Helen Henshaw, Dan Clark, Claire Falck, Sandra Smith

NIHR National Biomedical Research Unit in Hearing, Ropewalk House, 113 The Ropewalk, Nottingham NG5 1DU, UK; e-mail: S.Quinn@nottingham.ac.uk; helen.henshaw@nottingham.ac.uk

Received 17 June 2010, in revised form 24 September 2010

\begin{abstract}
We describe a task used to educate the public on the importance of hearing and the experience of hearing loss. During an open day at Nottingham University, participants were presented with verbal instructions (with or without a background masker) and asked to draw the image of a clock face as accurately as possible. Poorest performance was observed when the instructions were presented with a background masker. We suggest that the task could be applied effectively by healthcare professionals who aim to educate younger individuals of the difficulties associated with hearing loss.
\end{abstract}

Research has shown that speech-in-noise tasks are challenging to individuals with hearing loss (Lorenzi et al 2006). If people expose themselves to loud music, they could develop noise-induced hearing loss. This is important when we consider that young people regularly listen to music and may not appreciate the potential damage being caused to their hearing. It could be useful to educate the public on the risks of developing noise-induced hearing loss and enable people to understand the communication problems associated with hearing difficulties. We describe a pilot study and possible intervention which could be used within schools and universities to educate young people of the difficulties of understanding speech in noisy environments and potential risks they could face in developing noise-induced hearing loss.

Thirty-one participants aged between 28 and 64 years were recruited at an open day at Nottingham University. All of the participants reported normal hearing. The speech stimuli were recorded in a soundproof booth. To listen to these, see file 1 (instructions 1-5). Participants heard a list of 5 instructions over Sennheiser HD 25-1 II headphones via a laptop computer. In the masked conditions, the noise was presented at $75 \mathrm{~dB}$. In condition 1 (no masker), the speech was presented at $85 \mathrm{~dB}( \pm 5 \mathrm{~dB})$. In condition 2 (moderate difficulty) and 3 (high difficulty), the speech was presented at $82 \mathrm{~dB}( \pm 5 \mathrm{~dB})$ and $78 \mathrm{~dB}( \pm 5 \mathrm{~dB})$, respectively. Participants were randomly allocated to one of the three conditions: fourteen in condition 1 ; ten in condition 2 ; ten in condition 3. The participants were told that they would hear a list of 5 instructions over headphones that would require them to draw an image. Participants in the masked conditions were told that they would hear a rushing sound whilst listening to the instructions. They were asked to ignore this and focus on what the speaker told them to draw. Once the drawings were completed, they were given the opportunity to hear the instructions again in the presence of the masker (if they were allocated to the no-masker condition) or without the masker (if they were allocated to the mediumdifficulty or high-difficulty condition). This gave participants in the masked conditions the opportunity to hear the instructions clearly and observe their performance. Figure 1 (top of the figure) shows a representation of the clock participants should have produced and was shown to them at the end of the study. Participants completed the task in approximately $5 \mathrm{~min}$. 


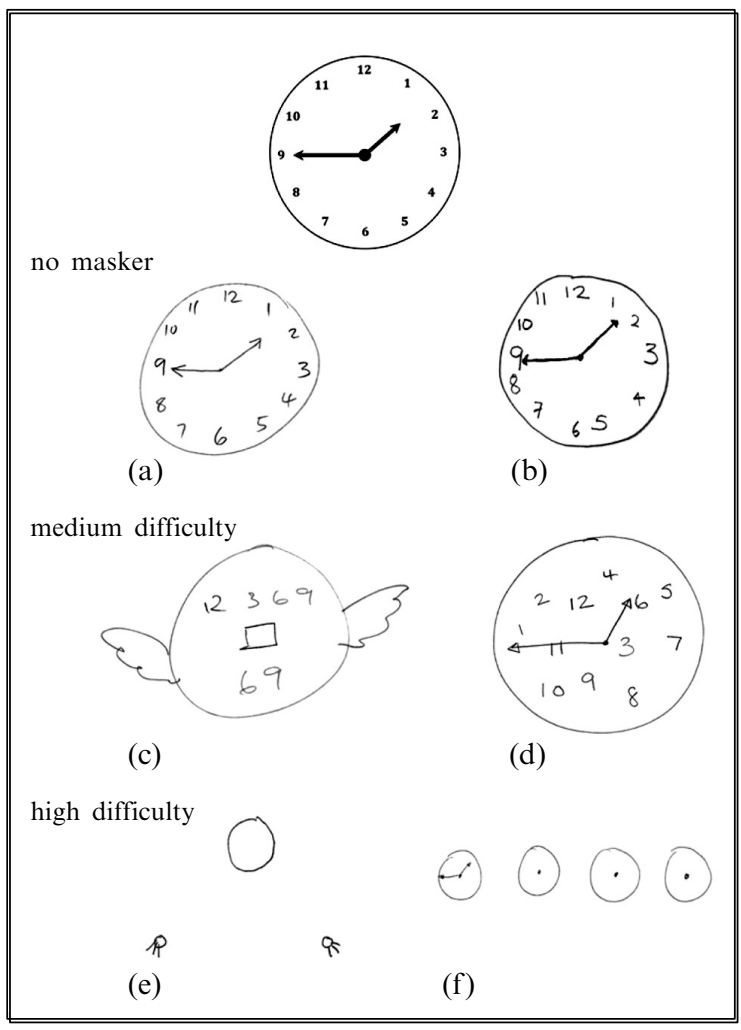

Figure 1. Examples of each drawing in the no-masker [(a) and (b)], medium-difficulty [(c) and (d)], and high-difficulty [(e) and (f)] conditions.

Examples of the clocks drawn in each condition are shown in figure 1. These examples show clear differences between conditions with some participants producing images where they had recognised the word 'hands', but failed to recognise that the image was a clock when the masker was presented (see figures 1c and 1e).

Scores were computed by two markers using a revised version of the scoring system of Royall et al (1998). A point was given for each item, and a score out of 16 was calculated for each drawing (see table 1 for the list of items in the scoring system). The markers (authors CF and SS) were not made aware of the true purpose of the study or method until they had completed the scores for each drawing. A mean score out of a maximum score of 16 for each drawing produced the following scores: in the no-masker condition, the mean score was $15.85(\mathrm{SD}=0.42)$; in the medium-difficulty condition, the mean score was 11.55 ( $\mathrm{SD}=4.08)$; in the high-difficulty condition the mean score was $7.95(\mathrm{SD}=7.95)$.

Analysis of the scoring system using a 'one-way' analysis of variance (speech condition with three levels, no masker, medium difficulty, and high difficulty) showed a significant difference between the three conditions $\left(F_{2,32}=15.16, p<0.05\right)$. A further a posteriori analysis, with a Bonferroni correction, showed that there was a significant difference between all three groups $(p<0.05)$. Therefore, the presence of the maskers affected the ability of listeners to correctly follow the instructions. Furthermore, the highdifficulty condition made it particularly difficult to follow the instructions in comparison to the medium-difficulty and no-masker conditions.

To assess performance for each instruction individually and to establish whether performance was poorer because participants had difficulty following a particular instruction, the list of items in the scoring system that assessed performance for each instruction was grouped together (see table 1). 
Table 1. Items scored for each instruction.

Instructions

Items used to score each instruction

(1 point for each item)

1. "Draw a large circle."

- drew a circle

- only 1 circle present

- circle is over 1 inch diameter

2. "Within the circle place the numbers $12,6,3$ and 9 as they would appear on the face of a clock."
- written one or more of the numbers 12 , 3,6 , and 9

- written all of the numbers $12,3,6$, and 9

- placed numbers in the correct location
3. "Now fill in the other numbers on the clock face."
- filled in some of the remaining numbers $(1,2,4,5,7,8,10,11)$

- filled in all of the remaining numbers $(1,2,4,5,7,8,10,11)$

- put remaining numbers in approximately the correct location
4. "Please place a dot in the centre of the circle."
- put a dot on the paper

- put a dot in the centre of the clock circle
5. "We would now like you to draw the hands of the clock and set the time to one forty-five. Make sure that each hand is drawn as an arrow."
- drew hands (of any kind, eg human hands or clock hands)

- drew clock hands

- drew clock hands as arrows

Figure 2 shows the percentage of attributes correctly drawn for each instruction and condition. If the no-masker condition showed poorer performance for any of the instructions provided to the participants, this would suggest that some of the instructions were ambiguous. However, this was not the case, with almost ceiling performance (average 95\%) in the no-masker condition. Given that performance in the masked conditions was poorer, the differences in performance between conditions can be attributed to the presence of the masker and not the instructions. Furthermore, performance decreases as a function of the level of the speech presented in each condition, with worst performance in the high-difficulty (lowest SNR) condition.

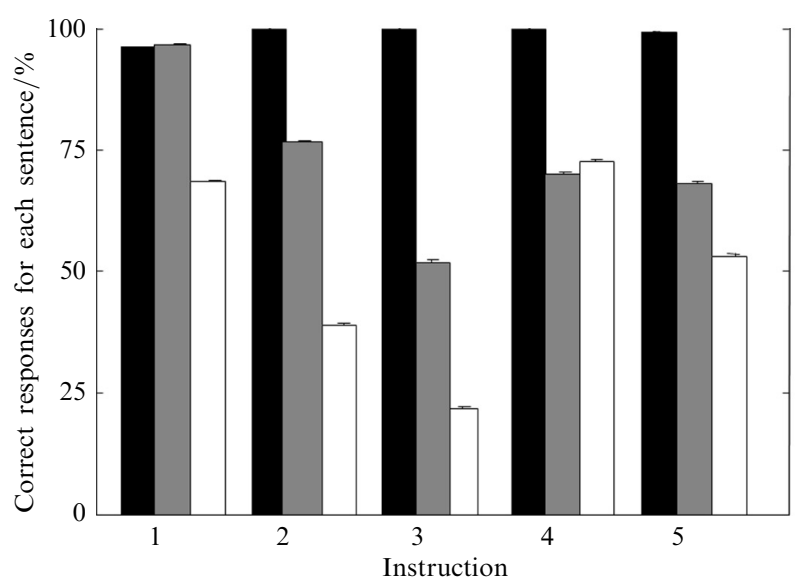

Condition

no masker

$\square$ moderate difficulty high difficulty

Figure 2. Percentage of correct attributes drawn for each instruction and in each condition with $95 \%$ confidence limits. 
In summary, we aimed to develop a task with the potential of informing the public about the importance of hearing and potential risks involved in developing noiseinduced hearing loss. The target population for this type of intervention would include children and teenagers. Younger children may not have developed the semantic abilities necessary to draw the complex object used in the current study. In order to successfully deliver this intervention to children, the images should be age-appropriate and familiar (eg draw a tree or house).

The simplicity of the task and scoring system suggest that it could be used in any educational setting. Particularly, teachers could adopt this task in classrooms or during undergraduate tutorials to explore the function and importance of the auditory system. Providing participants with the opportunity to hear the instructions again, once the task is complete, reinforces the inability to understand speech in noisy environments that has an immediate impact. Health organisations could adopt this task to reinforce the issues regarding hearing difficulties in noisy environments with the potential to change attitudes toward hearing loss.

Acknowledgments. This research was funded by the National Institute for Health Research. We would like to thank Heather Fortnum for her comments on earlier drafts of this paper.

\section{References}

Lorenzi C, Gilbert G, Carn H, Garnier S, Moore B C J, 2006 "Speech perception problems of the hearing impaired reflect inability to use temporal fine structure" Proceedings of the National Academy of Sciences of the USA $10318866-18869$

Royall D R, Cordes J A, Polk M, 1998 "CLOX: an executive clock drawing task" Journal of Neurology, Neurosurgery and Psychiatry with Practical Neurology $64588-594$ 


\section{PERTEPTION}

VOLUME 392010

www.perceptionweb.com

Conditions of use. This article may be downloaded from the Perception website for personal research by members of subscribing organisations. Authors are entitled to distribute their own article (in printed form or by e-mail) to up to 50 people. This PDF may not be placed on any website (or other online distribution system) without permission of the publisher. 